\title{
Study on Follicle Stimulating Hormone Receptor Gene Polymorphisms in South Indian Women with Polycystic Ovarian Syndrome
}

\author{
${ }^{1}$ Himavanth Reddy Kambalachenu, ${ }^{2}$ Solomon Franklin Durairaj Paul, \\ ${ }^{3}$ Sanjeeva Reddy Nellepalli and ${ }^{2}$ Perumal Venkatachalam \\ ${ }^{1}$ Department of Biomedical Sciences, College of Biomedical Science, \\ Technology and Research, Sri Ramachandra University, Chennai, India \\ ${ }^{2}$ Department of Human Genetics, College of Biomedical Science, \\ Technology and Research, Sri Ramachandra University, Chennai, India \\ ${ }^{3}$ Department of Reproductive Medicine, \\ Sri Ramachandra Medical College and Research Institute, Sri Ramachandra University, Chennai, India
}

Received 2013-01-06, Revised 2013-02-17; Accepted 2013-08-20

\begin{abstract}
Polycystic Ovarian Syndrome (PCOS) is the most common endocrine disorder of women in their reproductive ages. Though PCOS is a complex, heterogeneous disorder, but there is strong evidence for its genetic predisposition. The aim was to study the association of Follicle Stimulating Hormone Receptor (FSHR) gene polymorphisms rs1394205, rs6165 and rs6166 in south Indian women with PCOS. The present case control study includes 97 women with PCOS and 101 healthy women without any history of infertility. Polymerase chain reaction and Restriction fragment length polymorphism based method were applied to identify the genotypes. Distribution of alleles and genotypes did not differ significantly between PCOS and controls (p-value: $>0.05$ ). Genotypic association analysis shows a significant association of rs6166 (G/G) genotype with PCOS in recessive gene model (P value: 0.04). Haplotype frequencies and their association analysis did not show any significant difference between PCOS and controls. No strong linkage is observed between rs6165 and rs6166 in the present study. Our study reveals significant association of FSHR gene polymorphism, rs6166 with PCOS in recessive gene model. When we observe the genotype frequencies, high frequency of heterozygotes in the population shows that $\mathrm{rs} 6166(\mathrm{G} / \mathrm{A})$ in heterozygote condition is advantage to the population.
\end{abstract}

Keywords: FSHR, Polymorphism, Haplotype, Linkage Disequilibrium

\section{INTRODUCTION}

Polycystic Ovary Syndrome (PCOS) is an endocrine based infertility disorder affecting $4-8 \%$ of women in their reproductive ages globally (Moran et al., 2010). Hyperandrogenism is the hallmark of PCOS associated with abnormal menstrual cycles, polycystic ovaries, insulin resistance and altered pituitary gonadotropin secretions (Moran and Teede, 2009; Toulis et al., 2011).
Diagnosis of PCOS is primarily based on clinical and biochemical findings (Abbott et al., 2005). The first and systematic diagnostic criteria for PCOS was given in 1990 National Institute of Health (NIH) sponsored conference on PCOS, concluding clinical or biochemical evidence of androgen excess, chronic anovulation and the exclusion of other etiologies for diagnosis of PCOS. In 2003 ESHRE/ASRM-sponsored PCOS, Rotterdam Consensus Workshop Group, broadened the $1990 \mathrm{NIH}$

Corresponding Author: Perumal Venkatachalam, Department of Human Genetics, College of Biomedical Sciences, Technology and Research, Sri Ramachandra University, Chennai, India 
criteria by including the polycystic ovarian morphology (Rotterdam ESHRE/ASRM Sponsored PCOS Consensus Workshop Group, 2004). Rotterdam criteria for PCOS includes the presence of any two of the following features like clinical or biochemical evidence of androgen excess, oligo and/or anovulation and polycystic ovaries, excluding nonclassical congenital adrenal hyperplasia, Cushing syndrome, hyperprolactinemia and hypothyroidism. Although multiple genetic factors including mutations and polymorphisms of several genes have been associated with PCOS risk (LuqueRamirez et al., 2006), the data is inconsistent and further the complete mode of inheritance and the molecular genetic mechanisms underlying PCOS risk were yet to uncover. PCOS is associated with alterations in the function of the Hypothalamic-Pituitary-Gonadal (HPG) axis that may result from increased frequency of the hypothalamic Gonadotropin Releasing Hormone (GnRH) pulse generator. In healthy females high frequencies of $\mathrm{GnRH}$ pulses favors secretion of Luteinizing Hormone (LH) and lower pulses favors follicle stimulating hormone (FSH). HPG axis is thought to play a key role in PCOS patients with elevated serum LH levels and LH to FSH ratio suggesting an abnormal gonadotropin physiology (Taylor, 2006).

FSH plays an important role in maturation of ovarian follicles, dominant follicle selection in the ovary and aromatization of androgens. Dominant follicle selection is important for the reproductive function. FSH mediates its biological functions by binding through Follicle Stimulating Hormone Receptor (FSHR) expressed on Sertoli cells in testis and granulosa cells of the ovary (Wunsch et al., 2005). Animal studies using FSHR gene knockout mice showed cessation of folliculogenesis at preantral stage (Dierich et al., 1998), suggesting the importance of FSHR in ovarian physiology. Several polymorphisms have been identified in the FSHR gene, of these rs1394205 (A/G) polymorphism located in promoter (Wunsch et al., 2005) and in exon 10, two strongly linked rs6165 (A/G) and rs6166 (A/G) (Simoni et al., 1997). Polymorphism rs1394205 (A/A) genotype is associated with reduced expression of FSHR gene (Desai et al., 2011). FSHR gene polymorphism rs6166 (G/G) genotype in PCOS women is associated with amenorrhoea or anovulation (Sudo et al., 2002; Laven et al., 2003) and longer menstrual cycles (Greb et al., 2005) and hyperandrogenism (Valkenburg et al., 2009). High levels of serum FSH were found in PCOS patients with rs6166 $(\mathrm{G} / \mathrm{G})$ genotype proving the presence of this allele may be less sensitive to FSH (Valkenburg et al., 2009).
Pharmacogenetic studies revealed the varied response of FSHR gene polymorphisms to exogenous FSH hormone in patients undergoing Invitro fertilization (Mohiyiddeen and Nardo, 2010). Studies related to distribution of FSHR gene polymorphisms between the infertility subjects and their ethnic controls had shown inconsistent results (Sheikhha et al., 2011). In view of the inconclusive evidence regarding rs1394205, rs6165 and rs6166 genotype distribution in different ethnic populations, we conducted case-control genetic association by examining the allelic, genotype and haplotype association of FSHR gene polymorphisms in South Indian women with PCOS.

\section{MATERIALS AND METHODS}

The present genetic case-control study was approved by the Institutional ethics committee for human studies, Sri Ramachandra University (Ref. No: IECNI/08/Aug/05/32). The study subjects were recruited from SMART clinic, Department of Reproductive Medicine, Sri Ramachandra University. Selection of PCOS and healthy controls were based on 2003 Rotterdam criteria (PCOS could be diagnosed by two of the following three features: (1) oligo- or anovulation, (2) biochemical and/or clinical signs of hyperandrogenism like hirsutism, acne, male pattern baldness, facial and excess body hair or (3) presence of polycystic ovaries). Healthy women without any history of infertility and clinical signs of hyperandrogenism were included as controls. 97 women with PCOS and 101 healthy women were qualified to participate in the study. History of the subjects, demographic information and blood samples were collected from patients and controls after obtaining written consent.

\subsection{Genotyping Analysis}

Genomic DNA extraction from whole blood was carried out by using standard Phenol-chloroform method followed by ethanol purification, drying and then suspended in Tris-EDTA buffer and stored at $-80^{\circ} \mathrm{C}$. Genotyping was performed by Polymerase Chain Reaction (PCR) and Restriction Fragment Length Polymorphism (RFLP). Forward and reverse primer sequences, primer annealing temperatures, PCR product size are given in Table 1. Each PCR reaction was carried out in $20 \mathrm{uL}$ reaction, consisting of $10 \mathrm{uL}$ of 2X PCR master mix (Fermentas), $0.2 \mathrm{uL}(20$ picomoles) of forward primer, $0.2 \mathrm{uL}$ (20 picomoles) of reverse primer, 5.6 uL of MilliQ water and $4 \mathrm{uL}$ (200-400ng) of template DNA. 


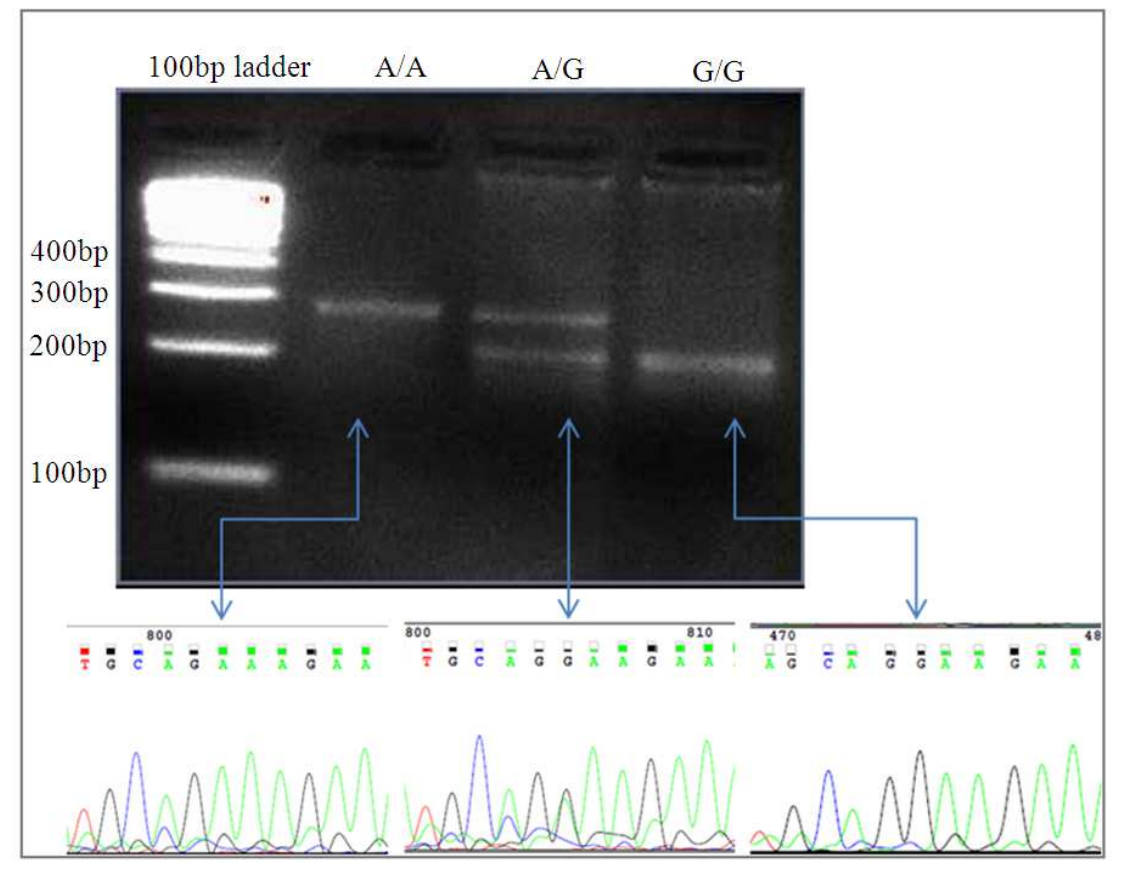

Fig. 1. rs1394205 polymorphism (A/G), \# rs1394205 polymorphism PCR product was digested with restriction enzyme MboII which gave an undigested product for A/A genotype (266bp), two fragments for G/G genotype (211 and 51bp) and three fragments for $\mathrm{A} / \mathrm{G}$ genotype $(266,211$ and $55 \mathrm{bp})$ and confirmed DNA sequencing

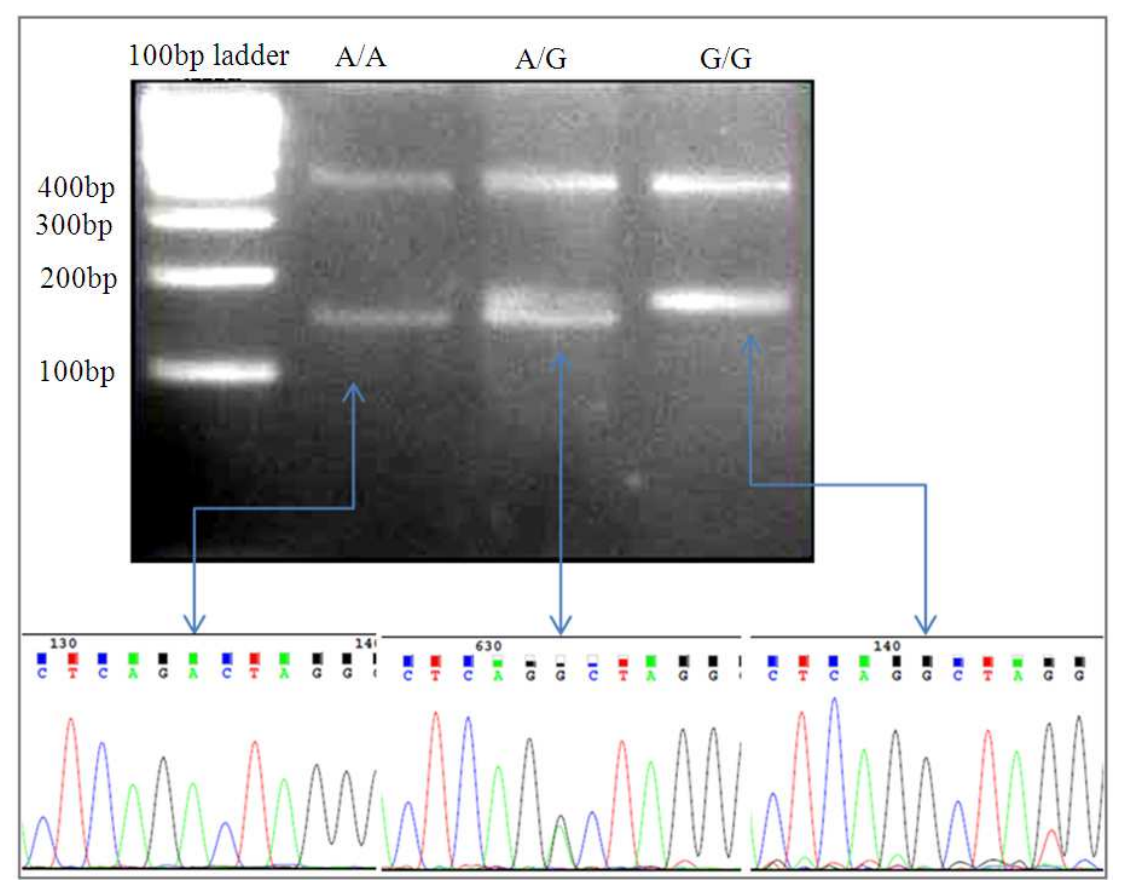

Fig. 2. rs6165 polymorphism (A/G), \# rs6165 polymorphism, PCR product was digested with Eam1105I gave rise to 3 fragments for the A/A genotype (403, 144 and 31bp), 2 fragments for $\mathrm{G} / \mathrm{G}$ genotype (403 and 175bp) and four fragments for A/G genotype $(403,175,144$ and $31 \mathrm{bp})$ and confirmation with DNA sequencing of PCR products 


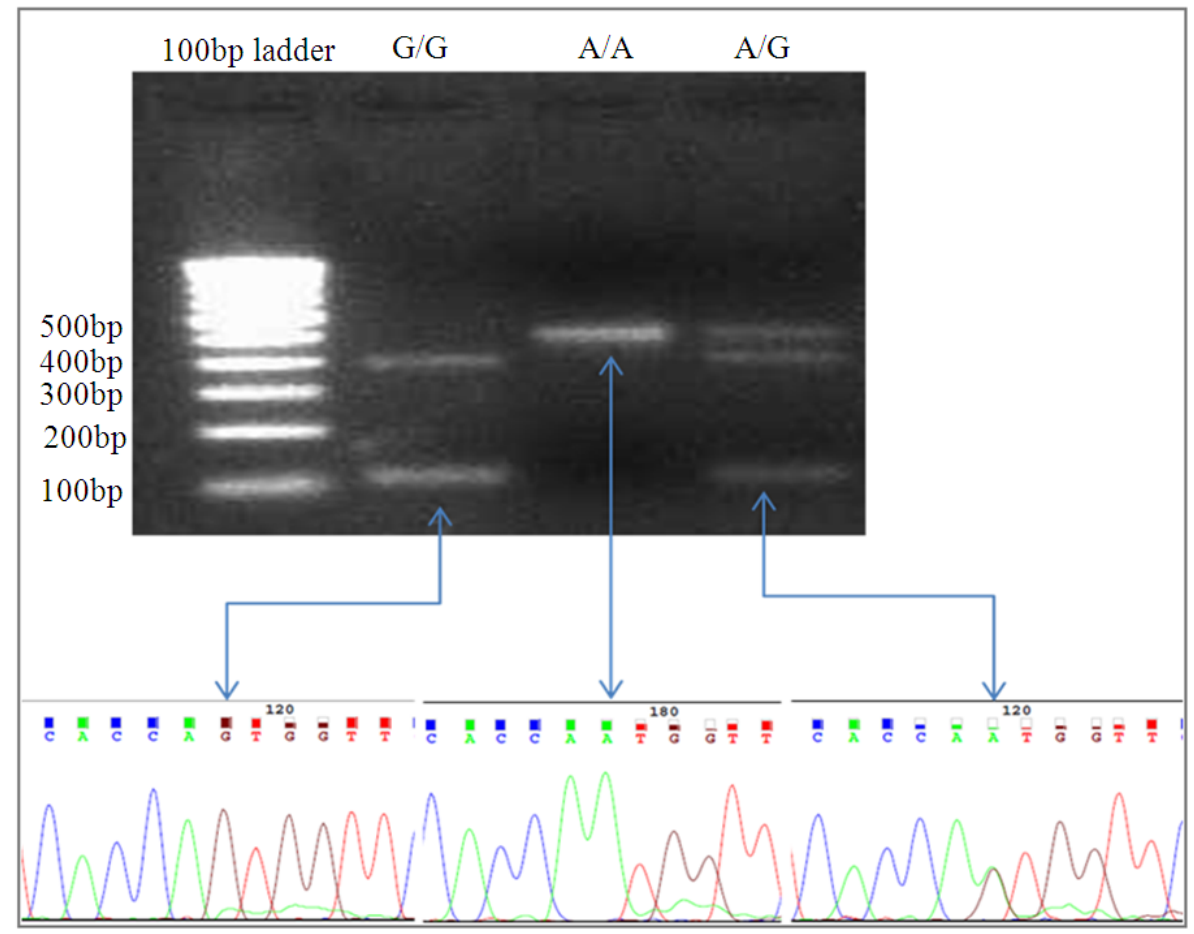

Fig. 3. rs6166 polymorphism (A/G), \# rs6166 polymorphisms was digested with BseNI gave an undigested product for A/A genotype (498bp), two fragments for G/G genotype (384 and 114bp) and three fragments for G/A genotypes (498, 384 and 114bp)

Table 1. PCR primer sequence, annealing temperatures (Tm.), PCR product size in base pairs (bp)

\begin{tabular}{lllll}
\hline Polymorphism & Primers & Tm. & Size & Reference \\
\hline rs1394205 & 5'-GGAGCTTCTGAGATCTGTGG-3' & $60.3^{\circ} \mathrm{C}$ & 266 & Gromoll et al. (2000) \\
\multirow{2}{*}{ rs6165 } & 5'-AAATGCCAGCCATGCAGTTG-3' & & & Sundblad et al. (2004) \\
\multirow{2}{*}{ rs6166 } & 5'-CCTGCACAAAGACAGTGATG-3' & $57.9^{\circ} \mathrm{C}$ & 578 & Present Study \\
& 5'-TGGCAAAGACAGTGAAAAAG-3' & & \multirow{2}{*}{498} & \\
\hline
\end{tabular}

PCR results were confirmed by agarose gel electrophoresis method. Restriction enzymes MboII (Fermentas), Eam1105I (Fermentas) and BseNI (Fermentas) are used for restriction digestion of rs1394205, rs6165 and rs6166 polymorphisms respectively and RFLP results were confirmed by DNA sequencing of selected samples (RFLP and DNA sequence images are provided as Fig. 1-3. in the Supplementary file 1).

\subsection{Statistical Analysis}

Statistical analysis for genotype data were done for all the polymorphisms in the present study. Plink 1.07 software (Purcell et al., 2007) is used to calculate allele and genotype distributions, Hardy-Weinberg
Equilibrium (HWE) deviations for PCOS and controls separately by exact tests. Single marker SNP association tests like allelic and genotypic (dominant and recessive) tests were done by using model option in plink 1.07 and haplotype frequencies and multi marker association test like haplotype association test to identify the risk haplotypes for PCOS. Haploview 4.2 was used to test the linkage disequilibrium analysis between the polymorphisms (Barrett et al., 2005).

\section{RESULTS}

All participants in the present study were in the age of $26.20 \pm 4.12$ (Mean \pm SD) in PCOS and $26.11 \pm 4.10($ Mean \pm SD) in controls. 
Table 2. Allele and genotype distribution, HWE of FSHR gene polymorphisms in PCOS and controls.

\begin{tabular}{|c|c|c|c|c|c|c|c|}
\hline \multirow[b]{2}{*}{ Polymorphism } & \multirow[b]{2}{*}{ Subjects } & \multicolumn{2}{|c|}{ Alleles } & \multicolumn{3}{|c|}{ Genotype counts (\%) } & \multirow[b]{2}{*}{ HWE P-value } \\
\hline & & A & $\mathrm{G}$ & $\mathrm{A} / \mathrm{A}$ & $\mathrm{A} / \mathrm{G}$ & $\mathrm{G} / \mathrm{G}$ & \\
\hline \multirow[t]{2}{*}{ rs1394205 } & Controls & 0.37 & 0.63 & $14(13.9)$ & $45(44.5)$ & $42(41.6)$ & 0.83 \\
\hline & PCOS & 0.35 & 0.65 & $16(16.5)$ & $36(37.1)$ & $45(46.4)$ & 0.07 \\
\hline \multirow[t]{2}{*}{ rs6165 } & Controls & 0.41 & 0.59 & $23(22.8)$ & 37 (36.6) & $41(40.6)$ & 0.01 \\
\hline & PCOS & 0.46 & 0.54 & $25(25.8)$ & $40(41.2)$ & $32(33.3)$ & 0.10 \\
\hline \multirow[t]{2}{*}{ rs6166 } & Controls & 0.56 & 0.44 & $31(30.7)$ & $52(51.5)$ & $18(17.8)$ & 0.69 \\
\hline & PCOS & 0.59 & 0.41 & $25(25.8)$ & $64(66.0)$ & $8(8.2)$ & 0.00 \\
\hline
\end{tabular}

Table 3. Association analysis of FSHR gene polymorphisms with PCOS.

\begin{tabular}{lllllll}
\hline Polymorphism & Minor allele & Major allele & Model & Chi-square & P-value & Odds Ratio (95\% CI) \\
\hline rs1394205 & A & G & Allelic & 0.05 & 0.82 & $0.95(0.63-1.43)$ \\
& & & Dominant & 0.46 & 0.49 & $0.95(0.64-1.41)$ \\
rs6165 & & & Recessive & 0.26 & 0.60 & $1.22(0.56-2.67)$ \\
& A & G & Allelic & 1.13 & 0.28 & $1.24(0.83-1.84)$ \\
& & & Dominant & 1.22 & 0.26 & $1.19(0.83-1.71)$ \\
rs6166 & & Recessive & 0.24 & 0.62 & $1.17(0.61-2.25)$ \\
& \multirow{2}{*}{ G } & A & Allelic & 0.21 & 0.63 & $0.90(0.61-1.35)$ \\
& & & Dominant & 0.59 & 0.44 & $0.88(0.56-1.38)$ \\
& & & Recessive & 3.97 & $0.04^{*}$ & $0.41(0.17-1.00)$ \\
\hline
\end{tabular}

Table 4. Haplotype frequencies and association analysis

\begin{tabular}{lllll}
\hline Haplotype $^{\mathrm{a}}$ & PCOS & Controls & Chi-square & P-value \\
\hline Omnibus & - & - & 5.45 & 0.60 \\
AAA & 0.10 & 0.12 & 0.29 & 0.58 \\
AAG & 0.08 & 0.09 & 0.05 & 0.81 \\
AGA & 0.10 & 0.07 & 1.16 & 0.28 \\
AGG & 0.07 & 0.08 & 0.41 & 0.52 \\
GAA & 0.14 & 0.10 & 1.54 & 0.21 \\
GAG & 0.14 & 0.11 & 1.22 & 0.26 \\
GGA & 0.25 & 0.28 & 0.44 & 0.50 \\
GGG & 0.12 & 0.16 & 1.09 & 0.29 \\
\hline
\end{tabular}

${ }^{\mathrm{a}}$ Haplotypes are in the order of rs1394205, rs6165 and rs6166

To study the rs1394205 polymorphism, PCR product was digested with restriction enzyme $M b o I I$ which gave an undigested product for A/A genotype (266bp), two fragments for $\mathrm{G} / \mathrm{G}$ genotype (211 and $51 \mathrm{bp}$ ) and three fragments for A/G genotype (266, 211 and 55bp). Similarly, for the rs6165 polymorphism, PCR product digested with Eam 1105I gave rise to 3 fragments for the A/A genotype (403, 144 and $31 \mathrm{bp}$ ), 2 fragments for $\mathrm{G} / \mathrm{G}$ genotype (403 and $175 \mathrm{bp}$ ) and four fragments for A/G genotype (403, 175, 144 and 31bp). For the rs6166 polymorphism, digestion with BseNI gave an undigested product for A/A genotype (498bp), two fragments for G/G genotype (384 and 114bp) and three fragments for G/A genotypes (498, 384 and 114bp).

Alleles, genotype frequencies and HWE deviations for FSHR gene polymorphisms rs1394205, rs6165 and rs6166 polymorphisms were given in Table 2 .
Distribution of Alleles and genotypes were not differed significantly between PCOS and controls. Deviations from HWE were checked for all polymorphisms in PCOS and controls separately. Polymorphism rs6165 in controls (p-value: 0.01 ) and rs6166 in PCOS (p-value: $<0.01$ ) were deviated from HWE. Heterozygote frequencies are more than the homozygotes particularly for rs6166 (Table 2).

Association analysis was performed based on allelic, dominant and recessive to identify the risk allele or genotype for PCOS (Table 3). Allelic and dominant model tests did not show any significant association with PCOS in tests except for rs6166 in recessive model. Polymorphism rs6166 found to be associated with PCOS in recessive model (chi-squared: 3.97 ; p-value: 0.04; Odds ratio (CI): 0.41 (0.17-1.00) (Table 3). Haplotype distribution and association analysis did not show any significance difference between PCOS and controls (Table 4). Five haplotypes GGA, GGG, GAG, GAA and AAA constitutes for more than $75 \%$ in both PCOS and controls. Linkage disequilibrium analysis was calculated by pairwise linkage analysis between rs6165 and rs6166 in both PCOS and controls together did not show strong linkage (D': 0.09 and r2: 0.008).

\section{DISCUSSION}

Endocrine and genetic studies of FSHR gene polymorphisms showed the association with different 
levels of serum FSH within the normal levels, duration of menstrual cycles and expression of FSHR transcripts, (Greb et al., 2005; Achrekar et al., 2010). Polymorphism rs6166 (G/G) is associated with high levels of FSH and followed by rs6166 (A/G) and rs6166 (A/A) genotype in PCOS (Valkenburg et al., 2009). Importance of FSHR in reproductive physiology and lack of allele and genotype distribution of FSHR gene polymorphisms and their association data in south Indian women with PCOS, we conducted a genetic case control study. Our data did not show any significant difference in distribution of allele or genotypes between PCOS and controls. In the present study we did not found any significant difference in frequency of rs1394205 polymorphism between PCOS and controls in contrast to earlier studies reported on Indonesian and German women undergoing ovarian hyperstimulation (Wunsch et al., 2005) and in women with amenorrhea in Indian population (Achrekar et al., 2010). Frequency of FSHR gene exon 10 polymorphisms rs6165 and rs6166 did not differed significantly with their ethnic controls and the results are similar to the previously reported studies based Han Chinese from Singapore (Tong et al., 2001) and Shanghai (Du et al., 2010), Japanese (Sudo et al., 2002), Dutch (Laven et al., 2003), Italian (Orio et al., 2006) Turkish (Unsal et al., 2009) and Caucasian (Valkenburg et al., 2009). The only significant association between PCOS and FSHR rs6166 $(\mathrm{G} / \mathrm{G})$ genotype was found in Korean women PCOS (Gu et al., 2010). Even in our study we found a significant association of $\operatorname{rs6166}(\mathrm{G} / \mathrm{G})$ genotype with PCOS in recessive model (Table 3).

A study on Han Chinese women with PCOS revealed the rs6165/rs6166 (G/A) haplotype of FSHR gene as a risk for PCOS (Du et al., 2010). In our study FSHR gene haplotype distribution not differed significantly between PCOS and controls and haplotype association analysis unable to find any risk haplotype (Table 4). FSHR gene polymorphisms influence basal FSH levels. Many studies independently tried to found the relation between serum FSH levels and FSHR gene polymorphisms. Polymorphism rs1394205 showed differing results without affecting basal FSH levels in women from Germany and Indonesian women undergoing In-vitro fertilization (Wunsch et al., 2005) and study from India showed the association with higher serum FSH levels in women with primary amenorrhea (Achrekar et al., 2010). Polymorphism rs6166 (G/G) genotype is found to be associated with high serum levels of FSH in women with PCOS but no significant association with PCOS risk (Valkenburg et al., 2009).
Several studies reported strong linkage disequilibrium between rs6165 and rs6166 in Caucasians (Valkenburg et al., 2009) and Japanese (Sudo et al., 2002) and few studies reported moderate to incomplete linkage between rs6165 and rs6166 reported in women with ovarian cancer (Yang et al., 2006). International HapMap Project website (http://hapmap.ncbi.nlm.nih.gov/) (International HapMap 3 Consortium, 2010) shows a strong linkage in populations from Central European ancestry (CEU) and no linkage in African ancestry in Southwest USA (ASW), Maasai in Kinyawa, Kenya (MKK), Luhya in Webuye from Kenya (LWK) and Yoruba in Ibadan (YIR) population from Nigeria. A recent study dedicated to linkage analysis in fertile normo-ovulatory group from Brazil found incomplete linkage between rs6165 and rs6166 (Rodini et al., 2011). Our results are in best agreement with the findings from Brazilian study.

\section{CONCLUSION}

In conclusion, our study reveals significant association of FSHR gene polymorphism, rs6166 with PCOS in genotypic association based on recessive model. When we observe the genotype frequencies, high frequency of heterozygotes in the population for rs6166 (51.5\% in controls and 66\% in PCOS) (Table 2) may reveal that the heterozygote condition is advantageous state like many other recessive genetic diseases. The rs1394205 and rs6166 polymorphisms did not show any significant association with PCOS risk. The present study has certain limitations due to confinding for three polymorphisms based on earlier works.

\section{ACKNOWLEDGEMENT}

We would like to extend sincere thanks to Dr V. Vettriselvi, Department of Human Genetics, Sri Ramachandra University, for her timely suggestions in preparation of this article. We are greatly thankful to Indian Council of Medical Research (ICMR), New Delhi for awarding Senior Research Fellowship (No: 3/1/2/12/2008$\mathrm{RHN}$ ) during the tenure of the present study.

\section{REFERENCES}

Abbott, D.H., D.K. Barnett, C.M. Bruns and D.A. Dumesic, 2005. Androgen excess fetal programming of female reproduction: A developmental aetiology for polycystic ovary syndrome. Hum. Reprod. Update, 11: 357-374. DOI: 10.1093/humupd/dmi013 
Achrekar, S.K., D.N. Modi, P.K. Meherji, Z.M. Patel and S.D. Mahale, 2010. Follicle stimulating hormone receptor gene variants in women with primary and secondary amenorrhea. J. Assist. Reprod. Genet., 27: 317-326. DOI: 10.1007/s10815-010-9404-9

Barrett, J.C., B. Fry, J. Maller and M.J. Daly, 2005. Haploview: Analysis and visualization of LD and haplotype maps. Bioinformation, 21: 263-265. DOI: 10.1093/bioinformatics/bth457

Desai, S.S., S.K. Achrekar, B.R. Pathak, S.K. Desai and V.S. Mangoli et al., 2011. Follicle-stimulating hormone receptor polymorphism $\left(\mathrm{G}^{-29} \mathrm{~A}\right)$ is associated with altered level of receptor expression in granulosa cells. J. Clin. Endocrin. Metab., 96: 2805-2812. DOI: 10.1210/jc.2011-1064

Dierich, A., M.R. Sairam, L. Monaco, G.M. Fimia and A. Gansmuller et al., 1998. Impairing FollicleStimulating Hormone (FSH) signaling in vivo: Targeted disruption of the FSH receptor leads to aberrant gametogenesis and hormonal imbalance. Proc. Natl. Acad. Sci. USA., 95: 13612-13617. DOI: 10.1073/pnas.95.23.13612

Du, J., W. Zhang, L. Guo, Z. Zhang and H. Shi et al., 2010. Two FSHR variants, haplotypes and metaanalysis in Chinese women with premature ovarian failure and polycystic ovary syndrome. Mol. Genet. Metab., 100: 292-295. DOI: 10.1016/j.ymgme.2010.03.018

Greb, R.R., K. Grieshaber, J. Gromoll, B. Sonntag and E. Nieschlag et al., 2005. A common single nucleotide polymorphism in exon 10 of the human follicle stimulating hormone receptor is a major determinant of length and hormonal dynamics of the menstrual cycle. J. Clin. Endocrinol. Metab., 90: 4866-4872. DOI: $10.1210 /$ jc. 2004-2268

Gromoll, J., M. Brocker, M. Derwahl and W. Hoppner, 2000. Detection of mutations in glycoprotein hormone receptors. Methods, 21: 83-97. DOI: 10.1006/meth.2000.0977

Gu, B.H., J.M. Park and K.H. Baek, 2010. Genetic variations of follicle stimulating hormone receptor are associated with polycystic ovary syndrome. Int. J. Mol. Med., 26: 107-112. DOI: 10.3892/ijmm_00000441

International HapMap 3 Consortium, 2010. Integrating common and rare genetic variation in diverse human populations. Nature, 467: 52-58. DOI: 10.1038 /nature09298
Laven, J.S., A.G. Mulders, D.A. Suryandari, J. Gromoll and E. Nieschlag et al., 2003. Follicle-stimulating hormone receptor polymorphisms in women with normogonadotropic anovulatory infertility. Fertil. Steril., 80: 986-992. DOI: 10.1016/S00150282(03)01115-4

Luque-Ramirez, M., J.L. San Millán and H.F. EscobarMorreale, 2006. Genomic variants in polycystic ovary syndrome. Clin. Chim. Acta., 366: 14-26. DOI: 10.1016/j.cca.2005.10.017

Mohiyiddeen, L. and L.G. Nardo, 2010. Singlenucleotide polymorphisms in the FSH receptor gene and ovarian performance: Future role in IVF. Hum. Fertil. (Camb)., 13: 72-78. DOI: 10.3109/14647271003632322

Moran, C., G. Tena, S. Moran, P. Ruiz and R. Reyna et al., 2010. Prevalence of polycystic ovary syndrome and related disorders in Mexican women. Gynecol. Obstet. Invest., 69: 274-280. DOI: 10.1159/000277640

Moran, L. and H. Teede, 2009. Metabolic features of the reproductive phenotypes of polycystic ovary syndrome. Hum. Reprod. Update, 15: 477-488. DOI: 10.1093/humupd/dmp008

Orio, F. Jr., E. Ferrarini, T. Cascella, A. Dimida and S. Palomba et al., 2006. Genetic analysis of the follicle stimulating hormone receptor gene in women with polycystic ovary syndrome. J. Endocrinol. Invest., 29: 975-982. PMID: 17259794

Purcell, S., B. Neale, K. Todd-Brown, L. Thomas and M.A. Ferreira et al., 2007. PLINK: A tool set for whole-genome association and population-based linkage analyses. Am. J. Hum. Genet., 81: 559-575. DOI: $10.1086 / 519795$

Rotterdam ESHRE/ASRM Sponsored PCOS Consensus Workshop Group, 2004. Revised 2003 consensus on diagnostic criteria and long-term health risks related to polycystic ovary syndrome. Fertil. Steril., 81: 1925. DOI: 10.1016/j.fertnstert.2003.10.004

Rodini, G.P., V.K. Genro, U. Matte, F.S. Pereira and J.P. Bilibio et al., 2011. There is no complete linkage between the polymorphisms N680S and T307A of the follicular stimulating hormone receptor gene in fertile women. J. Assist. Reprod. Genet., 28: 221224. DOI: $10.1007 / \mathrm{s} 10815-010-9503-7$

Sheikhha, M.H., M. Eftekhar and S. M. Kalantar, 2011. Investigating the association between polymorphism of follicle-stimulating hormone receptor gene and ovarian response in controlled ovarian hyperstimulation. J. Hum. Reprod. Sci., 4: 86-90. DOI: $10.4103 / 0974-1208.86089$ 
Simoni, M., J. Gromoll and E. Nieschlag, 1997. The follicle-stimulating hormone receptor: Biochemistry, molecular biology, physiology and pathophysiology. Endocr. Rev., 18: 739-773. DOI: 10.1210/er.18.6.739

Sudo, S., M. Kudo, S. Wada, O. Sato and A.J. Hsueh et al., 2002. Genetic and functional analyses of polymorphisms in the human FSH receptor gene. Mol. Hum. Reprod., 8: 893-899. DOI: 10.1093/molehr/8.10.893

Sundblad, V., V.A. Chiauzzi, M.E. Escobar, L. Dain, E.H. Charreau, 2004. Screening of FSH receptor gene in Argentine women with Premature Ovarian Failure (POF). Mol. Cell. Endocrinol., 222: 53-59. DOI: 10.1016/j.mce.2004.05.002

Taylor, A.E., 2006. Gonadotropin dysfunction in women with polycystic ovary syndrome. Fertil. Steril., 86 Suppl., $\quad 1$ : $\quad$ S12-S12. 10.1016/j.fertnstert.2006.05.001

Tong, Y., W.X. Liao, A.C. Roy and S.C. Ng, 2001. Absence of mutations in the coding regions of folliclestimulating hormone receptor gene in Singapore Chinese women with premature ovarian failure and polycystic ovary syndrome. Horm. Metab. Res., 33: 221-226. DOI: 10.1055/s-2001-14941

Toulis, K.A., D.G. Goulis, G. Mintziori, E. Kintiraki and E. Eukarpidis et al., 2011. Meta-analysis of cardiovascular disease risk markers in women with polycystic ovary syndrome. Hum. Reprod. Update, 17: 741-60. DOI: 10.1093/humupd/dmr025
Unsal, T., E. Konac, E. Yesilkaya, A. Yilmaz and A. Bideci et al., 2009. Genetic polymorphisms of FSHR, CYP17, CYP1A1, CAPN10, INSR, SERPINE1 genes in adolescent girls with polycystic ovary syndrome. J. Assist. Reprod. Genet., 26: 205216. DOI: $10.1007 / \mathrm{s} 10815-009-9308-8$

Valkenburg, O., A.G. Uitterlinden, D. Piersma, A. Hofman and A.P. Themmen et al., 2009. Genetic polymorphisms of $\mathrm{GnRH}$ and gonadotrophic hormone receptors affect the phenotype of polycystic ovary syndrome. Hum. Reprod., 24: 2014-2022. DOI: 10.1093/humrep/dep113

Wunsch, A., Y. Ahda, F. Banaz-Yaşar, B. Sonntag and E. Nieschlag et al., 2005. Single-nucleotide polymorphisms in the promoter region influence the expression of the human follicle-stimulating hormone receptor. Fertil. Steril., 84: 446-453. DOI: 10.1016/j.fertnstert.2005.02.031

Yang, C.Q., K.Y. Chan, H.Y. Ngan, U.S. Khoo and P.M. Chiu et al., 2006. Single nucleotide polymorphisms of follicle-stimulating hormone receptor are associated with ovarian cancer susceptibility. Carcinogenesis, 27: 1502-1506. DOI: 10.1093/carcin/bgl014 